\title{
Gestational Diabetes Mellitus and Folic Acid Supplementation in Pregnant Women: A Systematic Review
}

\author{
Stephanie Farah"1\#, Obey Albaini1" ${ }^{1 \#, ~ K a r l ~ J a l l a d ~}{ }^{2 *}$ \\ ${ }^{1}$ Lebanese American University Medical Center, Beirut, Lebanon \\ ${ }^{2}$ Obstetrics and Gynecology and Urology, Lebanese American University Medical Center, Beirut, Lebanon \\ Email: *karl.jallad@laumcrh.com
}

How to cite this paper: Farah, S., Albaini, O. and Jallad, K. (2021) Gestational Diabetes Mellitus and Folic Acid Supplementation in Pregnant Women: A Systematic Review. Journal of Diabetes Mellitus, 11, 26-39.

https://doi.org/10.4236/jdm.2021.111003

Received: December 14, 2020

Accepted: February 6, 2021

Published: February 9, 2021

Copyright $\odot 2021$ by author(s) and Scientific Research Publishing Inc. This work is licensed under the Creative Commons Attribution International License (CC BY 4.0).

http://creativecommons.org/licenses/by/4.0/

\begin{abstract}
Background: Gestational diabetes mellitus (GDM) is a common pregnancy disorder screened for between the $24^{\text {th }}$ and $28^{\text {th }}$ weeks of gestation using oral glucose tolerance test. GDM has maternal and fetal health implications. Objective: To assess the relation between folic acid supplementation in pregnant women and the risk of developing GDM. Search Strategy: The search employed topic-based strategies designed for each database in June 2020. Databases searched were Pubmed, Cochrane Library, Embase and Lebanese American University online database. Selection Criteria: Studies eligible were those targeting the association of GDM development and folic acid supplementation, including pregnant women who have developed GDM and pregnant women who were on folic acid supplementation and developed GDM. Both interventional and observational studies were included. Data Collection and Analysis: Two reviewers extracted the data independently. A third reviewer checked the data for consistency and clarity. Data extracted included the sample characteristics, sample size and outcomes. Cohen's $\kappa$ was used to assess agreement between reviewers. All tools and processes were piloted prior to use. Risk of bias was assessed using the Newcastle-Ottawa Scale. Data was presented in a tabulated form. Main Results: Six studies showed a proportional relation between folic acid intake and GDM, two reported a protective effect, and one cohort found no association. Conclusion: The inconsistent results made the formulation of a definitive conclusion difficult. Hence, larger studies are needed.
\end{abstract}

\section{Keywords}

Folic Acid, Gestational Diabetes

"Both are co-authors who contributed equally to the paper. 


\section{Introduction}

Gestational diabetes mellitus (GDM) is a common pregnancy disorder that is screened for between the $24^{\text {th }}$ and $28^{\text {th }}$ weeks of gestation, using oral glucose tolerance test (OGTT) [1] (Table 1 and Table 2). It is estimated that $6 \%$ of pregnancies in the United States are complicated by GDM [2]. Data clearly reflect that the rates of GDM in the US are lower in white women compared to other races and ethnicities [3]. Unfortunately, over the past few decades, the prevalence of GDM worldwide has increased [4], most likely due to elevated maternal age and body mass index (BMI) [5].

GDM increases the risk of preeclampsia, which was present in $9.8 \%$ of pregnant women with a fasting blood sugar (FBS) level less than $115 \mathrm{mg} / \mathrm{dL}$, but in $18 \%$ of women with FBS above $115 \mathrm{mg} / \mathrm{dL}$, as one study shows [6]. Similarly, in another study, the need for cesarean delivery was just $9.5 \%$ in controls who were not affected by GDM, but 17\% in women with diet-controlled GDM, and 25\% in patients who required anti-diabetic medication [7].

GDM does not only affect the maternal health status. The fetus is at an accentuated risk of neonatal hypoglycemia, hyperbilirubinemia, shoulder dystocia, traumatic delivery and stillbirth [8]. Alarming is the increased risk of developing maternal diabetes mellitus postpartum, with all its consequences on the overall mortality. This risk was described to be seven-times higher the general population in one study [9].

Table 1. Diagnostic values for gestational diabetes mellitus [1].

\begin{tabular}{|c|c|c|c|c|}
\hline \multirow{2}{*}{$\begin{array}{c}\text { System } \\
\text { Unit }\end{array}$} & \multicolumn{2}{|c|}{$\begin{array}{l}\text { Plasma or serum glucose level; carpenter } \\
\text { and coustan conversion }(\mathrm{mg} / \mathrm{dl})\end{array}$} & \multicolumn{2}{|c|}{$\begin{array}{l}\text { PLASMA level; national diabetes } \\
\text { data group conversion (mg/dl) }\end{array}$} \\
\hline & $\mathrm{mg} / \mathrm{dL}$ & $\mathrm{mmol} / \mathrm{L}$ & $\mathrm{mg} / \mathrm{dL}$ & $\mathrm{mmol} / \mathrm{L}$ \\
\hline Fasting & 95 & 5.3 & 105 & 5.8 \\
\hline 1 Hour & 180 & 10.0 & 190 & 10.6 \\
\hline 2 Hours & 155 & 8.6 & 165 & 9.2 \\
\hline 3 Hours & 120 & 7.8 & 145 & 8.0 \\
\hline
\end{tabular}

Table 2. International Association of Diabetes and Pregnancy Study Groups Recommendation the Diagnosis and Classification of Hyperglycemia in Pregnancy: one value equal or above to any of these values from the 75-g OGTT is enough for the diagnosis of GDM [26].

\begin{tabular}{ccc}
\hline \multirow{2}{*}{ Glucose measure } & \multicolumn{2}{c}{ Glucose concentration threshold } \\
\cline { 2 - 3 } & Mmol/L & $\mathrm{Mg} / \mathrm{dL}$ \\
\hline Fasting & 5.1 & 92 \\
1 Hour & 10.0 & 180 \\
2 Hours & 8.5 & 153 \\
\hline
\end{tabular}


Folic acid or vitamin $B_{9}$ is used as a supplement during the prenatal care, starting prior to conception, and to be continued throughout the first trimester, given that it decreases the risk of neural tube defects [10]. As recommendations clearly state the need for use of folic acid by pregnant women, the doses of this vitamin can reach 400 to $600 \mathrm{mcg}$ per day [11].

Folic acid interferes with metabolism, as some studies found that alterations in the ratio between folate and vitamin $\mathrm{B}_{12}$ can lead to fetal insulin resistance and increased risk of obesity [12]. Interestingly, several studies suggested that increased supplementation of folic acid imposed a higher risk of developing GDM [13]-[20]. However, no systematic review was ever published regarding folate use during pregnancy. Hence, the aim of this article is to assess the relation between the use of folic acid in pregnant women and the risk of developing GDM.

\section{Methods}

Design and methods used for this systematic review complied with the Preferred Reporting Items for Systematic Reviews and Meta-Analysis (PRISMA) guidelines. No review protocol was published. Eligibility criteria were based on the SPIDER guidelines. It is important to note that this is a systematic review that imposes no extra risk on patients as it just collected what was already published in the literature without further patient recruitment.

Studies eligible for this review were those targeting the association of GDM development and folic acid supplementation, including pregnant women who developed GDM and pregnant women who were on folic acid supplementation and developed GDM. Both interventional (randomized controlled trials) and observational studies (cohort, cross sectional, case control) were included. Qualitative, quantitative and mixed-methods research were searched for. The search employed topic-based strategies designed for each database in June 2020. There was no language or geographical restrictions.

Databases searched were Pubmed, the Cochrane Library, Medline and the Lebanese American University (LAU) online database. The search strategy included the study population and the phenomenon of interest using terms and keywords derived from scoping search and expertise in the subject field: pregnant, women, pregnancy, association, folic acid, folate, tetrahydrofolate, methenyltetrahydrofolate, folinic acid, folacine, pteroyglutamic acid, vitamin B9, complications, gestational diabetes mellitus.

Records were managed through a specific software while two reviewers (OA and SF) searched information sources independently and assessed identified studies for inclusion by grading each eligibility criterion as eligible, not eligible or might be eligible. The full text of a study was reviewed and the study considered potentially relevant, following discussion between the two reviewers, when it could not be clearly excluded on the basis of its title or abstract. Full text was obtained for abstracts with insufficient information or in a situation of disagreement. A study was included when both reviewers assessed it as satisfying the 
inclusion criteria. A third reviewer (KJ) mediated in the event of disagreement following discussion.

Using a standardized form, two reviewers (SF and OA) extracted the data independently. A third reviewer (KJ) checked the data for consistency and clarity. Data extracted included the following: sample characteristics, sample size and outcomes. Risk of bias for each included trial was independently assessed by the same initial reviewers. The third reviewer mediated in situations of disagreement. Cohen's $\kappa$ was used to assess agreement between reviewers. All tools and processes were piloted prior to use. Risk of bias was assessed using the Newcastle-Ottawa Scale (NOS).

Data was presented in a tabulated form to allow for semi qualitative comparison of study samples, associations and outcomes. All results were reported in the context of overall study quality.

It is unlikely that a meta-analysis could have been possible based on the findings that most of the retrieved studies are observational. The strength of the overall body of evidence was assessed using Grading of Recommendations, Assessment, Development and Evaluation (GRADE) (Appendix S1).

\section{Results}

\subsection{Search Results}

The search yielded 101 record hits. After removing duplicates, we ended up with 88 studies. We excluded 73 records during screening, with a remainder of 15 articles. After full article review, a total of 6 studies were excluded for various reasons (Table S2).

Finally, 9 articles were found to be relevant and met the inclusion criteria (Table S1). Most of them were observational. 7 were cohort [13] [14] [17] [19] [21] [22] [23], one had a case-control design [24] and only one was an interventional randomized controlled trial (RCT) [23]. 8 studies were conducted in China [13] [17] [19]-[24], and only one in USA [25]. All studies were published and written in English.

The eligible studies included a minimum of 326 pregnant women [14] and a maximum of 20,199 [25] with a mean of 5626 participants. Participants were diagnosed to have GDM based on different validated criteria: National diabetes data group and $3^{\text {rd }}$ International workshop conference [25], Carpenter Coustan [19] [24], International association of diabetes and pregnancy study groups [13] [14] [17] [21] [23].

\subsection{Individual Study Outcomes}

This review included one main primary outcome, which is the effect of folic acid (FA) supplementation on GDM risk.

\subsubsection{Studies Showing an Increased Risk of GDM with FA Supplementation}

The majority of the included studies showed a proportional relation between 
folic acid intake and GDM. Zhu et al. reported a proportional correlation between the risk of GDM and daily folic acid (FA) consumption during the first trimester (adjusted odds ratio (aOR) 2.25 [95\% CI 1.35 - 3.76]); in addition, pre-pregnancy body mass index (BMI) with folic acid play a role in increasing GDM risk (a pre-pregnancy BMI $>25 \mathrm{~kg} / \mathrm{m}^{2}$ with daily FA supplementation in the first trimester had a much higher risk of GDM (odds ratio OR $=5.63[95 \%$ CI 2.77 - 11.46]) compared to a pre-pregnancy BMI $<25 \mathrm{~kg} / \mathrm{m}^{2}$ and no FA supplements [19]. One study found that larger folate and potassium levels in the vitamin supplements used by pregnant ladies increased the risk of GDM [24]. It explained this finding by the hypothesis that higher FA level worsened the effects of vitamin B12 deficiency as well as insulin resistance. Innovatively, Zhang et al. demonstrated a synergistic effect of folic acid and pollution-induced GDM during the first trimester [22].

\subsubsection{Studies Targeting the Dosages and the Duration of Supplementation}

Some studies targeted the duration and the doses of FA supplementation. Cheng et al. proved that pre-pregnancy FA supplementation for a duration of 3 months or longer was associated with an increased risk of GDM (adjusted relative risk (aRR): 1.72; 95\% CI: 1.17 - 2.53) [13]. Another study showed an increase risk of GDM with both higher doses and longer duration. Short duration was defined as taking FA in the pre pregnancy period for less than 4 weeks and/or less than 16 weeks during pregnancy but before OGTT, and long duration defined as starting supplements for at least 4 weeks and continued for at least 16 weeks during pregnancy before OGTT [17]. The following 4 groups were studied: FA400-S (400 - $800 \mathrm{mcg} /$ day short duration), FA400-L (400 - $800 \mathrm{mcg} /$ day long duration), FA800-S ( $>800 \mathrm{mcg} /$ day short duration), and FA800-L ( $>800 \mathrm{mcg} /$ day for long duration). Results showed an odds ratio OR with 95\% CIs of GDM of 1.23 (0.84 - 1.80), 1.23 (0.78 - 1.94), 1.37 (0.93 - 2.01), and 2.36 (1.51 - 3.69) for FA400-S, FA400-L, FA800-S and FA800-L, respectively, compared with non-users. After adjusting for confounders, those with FA800-L still had significant higher risk of GDM (adjusted OR [95\% CI] 2.09 [1.30 - 3.36]). Similarly, Huang et al. showed that a longer duration of FA ( $>90$ days) increases GDM risk (adjusted OR 3.45 (95\% CI: $1.01,11.8)$ compared to a shorter duration [14].

\subsubsection{Studies Showing a Decreased Risk of GDM with FA Supplementation}

On the other hand, 2 studies reported a protective effect of folic acid on GDM risk. One showed that women with adequate total folate intake ( $>400 \mathrm{mg} / \mathrm{day}$ ) had a RR of GDM of 0.83 (95\% CI 0.72, 0.95, P = 0.007) compared with women with a lesser intake (<400 mg/day). The RRs of GDM for 1 - 399, $400-599$, and $>600 \mathrm{mg} /$ day of supplemental folate intake were $0.83,0.77$, and 0.70 , respectively, compared with no supplemental folate intake $(\mathrm{P}=0.002)$. The association between supplemental folate intake and GDM risk largely persisted after additional adjustment for intake of multivitamins and other micronu- 
trients, as well as among women who likely planned for the pregnancy [17]. Interestingly, another study demonstrated that an individualized supplementation of folic acid according to the polymorphisms of methylene terahydrofolate reductase (MTHFR) and methionine synthase reductase (MTTR) can reduce the GDM risk [23].

\subsubsection{Studies Showing no Relation between the Risk of GDM and FA Supplementation}

Finally, one cohort study found no statistical significance between GDM and folic acid supplementation after adjustment for rs1801133 polymorphisms [21]. Higher RBC folate, partly caused by MTHFR 677C $\rightarrow \mathrm{T}$, may be associated with increased GDM risk, even in early pregnancy; participants with the TT genotype had the highest RBC folate concentrations. Those with heterozygous or homozygous variants did not have a significantly higher risk of GDM than did women with C alleles.

\section{Discussion}

\section{Main Findings}

Out of the 9 studies included in this review, 6 supported the association between increased folic acid intake and the development of GDM during pregnancy, 2 showed an inverse relation between folate supplementation and GDM risk, while just one found no association at all when several factors were adjusted for, including genetics [21]. However, in the last study, the sample size was small compared to others $(n=336)$, and it focused on one ethnicity in China. Hence, further studies on different ethnicities and higher sample sizes are needed to prove the lack of relation between the risk of GDM during pregnancy and FA supplementation when C677T polymorphism in the MTHFR gene is considered. This requires wide genetic studies to link the different alleles of the MTHFR gene with the risk of developing GDM and use of FA supplements, as another study that investigated the A1298C allele of MTHFR gene found an inverse relation between FA supplementation and GDM risk [23]. However, applying genetic studies necessitates the assessment for cost-effectiveness and the identification of the high-risk population which could benefit from genetic detection of the different MTHFR alleles.

One very large study with 20,199 pregnancies supported the inverse correlation between FA supplementation and GDM development [25]. It included the highest number of participants (35.5\% of patients in this review). The enormous sample size gives more credibility to the results, given that this study accounted for the different confounding factors (physical activity and different dietary lifestyles). Nonetheless, the fact that some participants were self-reporting their FA intake definitely increased the risk of bias. In addition, the group we would be interested in was small, as $4.1 \%$ of the patients developed GDM. Hence, we need a similar huge study but with a case-control design where more GDM cases can be recruited. 
Chen Q. et al. focused on multivitamin supplementation [25], yet there is a need to identify the overall intake of FA including diet. Zhang et al. studied air pollution and GDM [22]. Their results raised concerns for pregnant women living in big cities, and thus, further studies are warranted to link the level of contamination in the air to the accepted doses of FA during pregnancy.

The novel cohort study suggesting that a daily FA dose of $800 \mathrm{mg}$ increased the risk of GDM can push towards a revision of the recommendations of daily doses higher than $400 \mathrm{mg}$ [17]. Two studies discussing the duration of use of FA [13] [14] also proved the need for folic acid supplementation as it not only can decrease the risk of neural tube defects, but also can decrease the risk of GDM compared to no use; however, this vitamin should not be taken for more than 3 months prior to conception [13] and for a duration less than 90 days post-conception [14]. It is important to note that diet alone was not sufficient in providing the needed levels of micronutrients for pregnant women, including folate which was scarcely taken from food, as shown by Kozlowska A. [26] and Looman M. [27].

Zhu et al. suggested that increased FA intake among pregnant ladies can lead to GDM directly by explaining that more folate can augment the effects of vitamin B12 deficiency, most likely focusing on insulin resistance, or indirectly via the harmful effects of non-metabolized folate [19]. Understanding the mechanism behind this process can be reached through other studies targeting the metabolism of folate.

\section{Interpretation}

The analysis of the data in these studies combined cannot reach a conclusion on whether or not FA supplementation pre-pregnancy and during gestation increase the risk of developing GDM, as the results are inconsistent.

Aside from the articles discussed in this review, one study showed that babies born to women with GDM had higher cord plasma concentration of folic acid, which it attributed to an altered feto-placental metabolism of the vitamin or to a change in the transport mechanism [28]. Another study found that pregnant women with GDM had higher folate levels in the plasma [29].

In one article, it was shown that higher red blood cell (RBC) folate concentrations were associated with higher GDM risk, with a relative risk RR per 1 standard deviation (1-SD) increase in RBC folate level equal to 1.16 (1.03 - 1.30) [20].

Folate and vitamin $\mathrm{B}_{12}$ are essential factors in the metabolism of homocysteine. Interestingly, Guvenet al. in 2006 found no statistical difference between women with GDM and normal controls concerning blood folate level, but blood homocysteine levels were significantly higher among GDM women $(9.0 \pm 3.1 \mu \mathrm{mol} / \mathrm{l})$ compared to normal pregnant ladies $(7.4 \pm 1.6 \mu \mathrm{mol} / \mathrm{l})[30]$.

\section{Strengths and Limitations}

This is the first systematic review to be conducted on such a topic. Several stu- 
dies were contrived very recently to understand the correlation between FA supplementation for pregnant women and GDM risk, making the whole idea a hot topic in 2020, knowing that FA supplementation in specific doses is recommended starting preconception to decrease the risk of neural tube defects. Hence, once more data are available and a clear conclusion can be drawn, FA supplementation guidelines during pregnancy might be subject to change.

However, this systematic review has several limitations. First, just one randomized controlled trial was included, while the rest of the studies were observational. Not all studies were able to specify dose and duration ranges. In addition, FA supplementation from food could not be clearly assessed. Most of the observational studies were based on questionnaires or medical records, which impose a high risk of bias. 8 out of 9 studies were conducted in China, making any results driven from these studies only applicable on the Chinese population until replicates are made on other populations. Finally, the diagnostic criteria for GDM and the food frequency questionnaire used were different among the studies. This acted as a limitation for comparing the different results from similar studies discussed in this review.

\section{Conclusion}

The inconsistent results from the different articles discussed in this review make the formulation of a definitive conclusion on the relation between GDM and FA supplementation in pregnancy difficult. Hence, larger studies, consisting of different ethnicities, and applying the same criteria for GDM diagnosis are needed to be able to draw a conclusion. In addition, the dosage and duration of FA supplementation should be assessed away from subjective questionnaires and regardless of dietary FA intake, as studies have shown that FA intake from food does not suffice the needs of pregnancy.

\section{Contribution to Authorship}

$\mathrm{OA}, \mathrm{SF}$ and KJ conceived and designed the study. OA and SF performed the literature search and data extraction. KJ checked the data. OA and SF performed the data analysis. OA, SF and KJ wrote the article.

\section{Conflicts of Interest}

The authors declare no conflicts of interest regarding the publication of this paper.

\section{References}

[1] American Diabetes Association (2018) Management of Diabetes in Pregnancy: Standards of Medical Care in Diabetes. Diabetes Care, 41, S137-S143. https://doi.org/10.2337/dc18-S013

[2] Deputy, N.P., Kim, S.Y., Conrey, E.J. and Bullard, K.M. (2018) Prevalence and Changes in Preexisting Diabetes and Gestational Diabetes among Women Who Had a Live Birth-United States, 2012-2016. Morbidity and Mortality Weekly Re- 
port, 67, 1201-1207. https://doi.org/10.15585/mmwr.mm6743a2

[3] Ferrara, A. (2007) Increasing Prevalence of Gestational Diabetes Mellitus: A Public Health Perspective. Diabetes Care, 30, S141-S146. https://doi.org/10.2337/dc07-s206

[4] Simmons, D. (2011) Diabetes and Obesity in Pregnancy. Best Practice \& Research Clinical Obstetrics \& Gynaecology, 25, 25-36. https://doi.org/10.1016/j.bpobgyn.2010.10.006

[5] Feig, D.S., Hwe, J., Shah, B.R., Booth, G.L., Bierman, A.S. and Lipscombe, L.L. (2014) Trends in Incidence of Diabetes in Pregnancy and Serious Perinatal Outcomes: A Large, Population-Based Study in Ontario, Canada, 1996-2010. Diabetes Care, 37, 1590-1596. https://doi.org/10.2337/dc13-2717

[6] Yogev, Y., Xenakis, E.M.J. and Langer, O. (2020) The Association between Preeclampsia and the Severity of Gestational Diabetes: The Impact of Glycemic Control. American Journal of Obstetrics and Gynecology, 191, 1655-1660. https://pubmed.ncbi.nlm.nih.gov/15547538

[7] Ehrenberg, H.M., et al. (2004) The Influence of Obesity and Diabetes on the Risk of Cesarean Delivery. American Journal of Obstetrics and Gynecology, 191, 969-974. https://pubmed.ncbi.nlm.nih.gov/15467574

[8] Rosenstein, M.G., Cheng, Y.W., Snowden, J.M., Nicholson, J.M. and Caughey, A.B. (2012) Risk of Stillbirth and Infant Death Stratified by Gestational Age. Obstetrics \& Gynecology, 120, 76-82. https://doi.org/10.1097/AOG.0b013e31825bd286

[9] Jiwani, A., Marseille, E., Lohse, N., Damm, P., Hod, M. and Kahn, J.G. (2012) Gestational Diabetes Mellitus: Results from a Survey of Country Prevalence and Practices. Journal of Maternal-Fetal and Neonatal Medicine, 25, 600-610. https://doi.org/10.3109/14767058.2011.587921

[10] Imbard, A., Benoist, J.-F. and Blom, H.J. (2013) Neural Tube Defects, Folic Acid and Methylation. International Journal of Environmental Research and Public Health, 10, 4352-4389. https://doi.org/10.3390/ijerph10094352

[11] Castaño, E., Piñuñuri, R., Hirsch, S. and Ronco, A.M. (2017) Folate and Pregnancy, Current Concepts: It Is Required Folic Acid Supplementation? Revista Chilena de Pediatría, 88, 199-206. https://doi.org/10.4067/S0370-41062017000200001

[12] Yajnik, C.S., Deshpande, S.S., Jackson, A.A., Refsum, H., Rao, S., Fisher, D.J., et al. (2008) Vitamin B12 and Folate Concentrations during Pregnancy and Insulin Resistance in the Offspring: The Pune Maternal Nutrition Study. Diabetologia, 51, 29-38. https://doi.org/10.1007/s00125-007-0793-y

[13] Cheng, G., Sha, T., Gao, X., He, Q., Wu, X., Tian, Q., et al. (2019) The Associations between the Duration of Folic Acid Supplementation, Gestational Diabetes Mellitus, and Adverse Birth Outcomes Based on a Birth Cohort. International Journal of Environmental Research and Public Health, 16, 4511.

https://doi.org/10.3390/ijerph16224511

[14] Huang, L., Yu, X., Li, L., Chen, Y., Yang, Y., Yang, Y., et al. (2019) Duration of Periconceptional Folic Acid Supplementation and Risk of Gestational Diabetes Mellitus. Asia Pacific Journal of Clinical Nutrition, 28, 321-329.

[15] Krishnaveni, G.V., Hill, J.C., Veena, S.R., Bhat, D.S., Wills, A.K., Karat, C.L.S., et al. (2009) Low Plasma Vitamin B12 in Pregnancy Is Associated with Gestational "Diabesity" and Later Diabetes. Diabetologia, 52, 2350-2358. https://doi.org/10.1007/s00125-009-1499-0

[16] Lai, J.S., Pang, W.W., Cai, S., Lee, Y.S., Chan, J.K.Y., Shek, L.P.C., et al. (2018) High Folate and Low Vitamin B12 Status during Pregnancy Is Associated with Gestational Diabetes Mellitus. Clinical Nutrition, 37, 940-947. 
https://doi.org/10.1016/j.clnu.2017.03.022

[17] Li, Q., Zhang, Y., Huang, L., Zhong, C., Chen, R., Zhou, X., et al. (2019) High-Dose Folic Acid Supplement Use from Prepregnancy through Midpregnancy Is Associated with Increased Risk of Gestational Diabetes Mellitus: A Prospective Cohort Study. Diabetes Care, 42, e113-e115. https://doi.org/10.2337/dc18-2572

[18] Li, S., Hou, Y., Yan, X., Wang, Y., Shi, C., Wu, X., et al. (2019) Joint Effects of Folate and Vitamin B12 Imbalance with Maternal Characteristics on Gestational Diabetes Mellitus. Journal of Diabetes, 11, 744-751. https://doi.org/10.1111/1753-0407.12899

[19] Zhu, B., Ge, X., Huang, K., Mao, L., Yan, S., Xu, Y., et al. (2016) Folic Acid Supplement Intake in Early Pregnancy Increases Risk of Gestational Diabetes Mellitus: Evidence from a Prospective Cohort Study. Diabetes Care, 39, e36-e37. https://doi.org/10.2337/dc15-2389

[20] Xie, K., Xu, P., Fu, Z., Gu, X., Li, H., Cui, X., et al. (2019) Association of Maternal Folate Status in the Second Trimester of Pregnancy with the Risk of Gestational Diabetes Mellitus. Food Science \& Nutrition, 7, 3759-3765.

https://doi.org/10.1002/fsn3.1235

[21] Liu, P., Liu, Y., Ma, L., Yao, A.M., Chen, X.Y., Hou, Y.X., et al. (2020) Associations between Gestational Diabetes Mellitus Risk and Folate Status in Early Pregnancy and MTHFR C677T Polymorphisms in Chinese Women. Diabetes, Metabolic Syndrome and Obesity, 13, 1499-1507. https://doi.org/10.2147/DMSO.S250279

[22] Zhang, H., Dong, H., Ren, M., Liang, Q., Shen, X., Wang, Q., et al. (2020) Ambient Air Pollution Exposure and Gestational Diabetes Mellitus in Guangzhou, China: A Prospective Cohort Study. Science of the Total Environment, 699, Article ID: 134390. https://doi.org/10.1016/j.scitotenv.2019.134390

[23] Li, X., Jiang, J., Xu, M., Xu, M., Yang, Y., Lu, W., et al. (2015) Individualized Supplementation of Folic Acid According to Polymorphisms of Methylenetetrahydrofolate Reductase (MTHFR), Methionine Synthase Reductase (MTRR) Reduced Pregnant Complications. Gynecologic and Obstetric Investigation, 79, 107-112. https://doi.org/10.1159/000367656

[24] Chen, Q., Feng, Y., Yang, H., Wu, W., Zhang, P., Wang, K., et al. (2019) A Vitamin Pattern Diet Is Associated with Decreased Risk of Gestational Diabetes Mellitus in Chinese Women: Results from a Case Control Study in Taiyuan, China. Journal of Diabetes Research, 2019, Article ID: 5232308. https://doi.org/10.1155/2019/5232308

[25] Li, M., Li, S., Chavarro, J.E., Gaskins, A.J., Ley, S.H., Hinkle, S.N., et al. (2019) Prepregnancy Habitual Intakes of Total, Supplemental, and Food Folate and Risk of Gestational Diabetes Mellitus: A Prospective Cohort Study. Diabetes Care, 42, 1034-1041. https://doi.org/10.2337/dc18-2198

[26] Kozlowska, A., Jagielska, A.M., Okreglicka, K.M., Dabrowski, F., Kanecki, K., Nitsch-Osuch, A., et al. (2018) Dietary Vitamin and Mineral Intakes in a Sample of Pregnant Women with Either Gestational Diabetes or Type 1 Diabetes Mellitus, Assessed in Comparison with Polish Nutritional Guidelines. Ginekologia Polska, 89, 581-586. https://doi.org/10.5603/GP.a2018.0100

[27] Looman, M., Schoenaker, D.A.J.M., Soedamah-Muthu, S.S., Mishra, G.D., Geelen, A. and Feskens, E.J.M. (2019) Pre-Pregnancy Dietary Micronutrient Adequacy Is Associated with Lower Risk of Developing Gestational Diabetes in Australian Women. Nutrition Research, 62, 32-40. https://doi.org/10.1016/j.nutres.2018.11.006

[28] Barzilay, E., Moon, A., Plumptre, L., Masih, S.P., Sohn, K.-J., Visentin, C.E., et al. (2018) Fetal One-Carbon Nutrient Concentrations May Be Affected by Gestational Diabetes. Nutrition Research, 55, 57-64. 
https://doi.org/10.1016/j.nutres.2018.04.010

[29] Berglund, S.K., García-Valdés, L., Torres-Espinola, F.J., Segura, M.T., Martínez-Zaldívar, C., Aguilar, M.J., et al. (2016) Maternal, Fetal and Perinatal Alterations Associated with Obesity, Overweight and Gestational Diabetes: An Observational Cohort Study (PREOBE). BMC Public Health, 16, 207. https://doi.org/10.1186/s12889-016-2809-3

[30] Guven, M.A., Kilinc, M., Batukan, C., Ekerbicer, H.C. and Aksu, T. (2006) Elevated Second Trimester Serum Homocysteine Levels in Women with Gestational Diabetes Mellitus. Archives of Gynecology and Obstetrics, 274, 333-337.

https://doi.org/10.1007/s00404-006-0191-6 


\section{Appendix S1: Study Design}

Design and methods used for this systematic review complied with the Preferred Reporting Items for Systematic Reviews and Meta-Analysis (PRISMA) guidelines. Eligibility criteria were based on the SPIDER guidelines. It is important to note that this is a systematic review that imposes no extra risk on patients as it just collected what was already published in the literature without further patient recruitment.

Studies eligible for this review were those targeting the association of GDM development and folic acid supplementation, including pregnant women who developed GDM and pregnant women who were on folic acid supplementation and developed GDM. Both interventional (randomized controlled trials) and observational studies (cohort, cross sectional, case control) were included. Qualitative, quantitative and mixed-methods research were searched for. The search employed topic-based strategies designed for each database in June 2020. There was no language or geographical restrictions. Databases searched were Pubmed, the Cochrane Library, Medline and the Lebanese American University (LAU) online database. The search strategy included the study population and the phenomenon of interest using terms and keywords derived from scoping search and expertise in the subject field: pregnant, women, pregnancy, association, folic acid, folate, tetrahydrofolate, methenyltetrahydrofolate, folinic acid, folacine, pteroyglutamic acid, vitamin $\mathrm{B}$, complications, gestational diabetes mellitus.

Records were managed through a specific software while two reviewers (OA and SF) searched information sources independently and assessed identified studies for inclusion, facilitated by grading each eligibility criterion as eligible, not eligible or might be eligible. The full text of a study was reviewed and the study considered potentially relevant, following discussion between the two reviewers, when it could not be clearly excluded on the basis of its title or abstract. Full text was obtained for abstracts with insufficient information or in a situation of disagreement. A study was included when both reviewers assessed it as satisfying the inclusion criteria. A third reviewer (KJ) mediated in the event of disagreement following discussion.

Using a standardized form, two reviewers (SF/OA) extracted the data independently. A third reviewer (KJ) checked the data for consistency and clarity. Data extracted included the following: sample characteristics, sample size and outcomes. Risk of bias for each included trial was independently assessed by the same initial reviewers. The third reviewer mediated in situations of disagreement. Cohen's $\kappa$ was used to assess agreement between reviewers. All tools and processes were piloted prior to use. Risk of bias was assessed using the Newcastle-Ottawa Scale (NOS).

Data was presented in a tabulated form to allow for semi qualitative comparison of study sample, association and outcome. All results were reported in the context of overall study quality. It is unlikely that a meta-analysis could have been possible based on the findings that most of the retrieved studies are observational. The strength of the overall body of evidence was assessed using Grading of Recommendations, Assessment, Development and Evaluation (GRADE). 
Table S1. Included studies.

\begin{tabular}{|c|c|c|c|c|}
\hline $\begin{array}{l}\text { First Author's } \\
\text { Name }\end{array}$ & $\begin{array}{c}\text { Year/Country/ } \\
\text { Study design }\end{array}$ & Sample size & Inclusion/Exclusion Criteria & Results \\
\hline Chen, Q. & $\begin{array}{l}\text { 2019/China/ } \\
\text { Case-Control }\end{array}$ & $\begin{array}{l}\text { 9556: } 1464(15.3 \%) \\
\text { GDM cases and } 8092 \\
(84.7 \%) \text { non-GDM } \\
\text { controls }\end{array}$ & $\begin{array}{c}\text { Inclusion criteria: women aged } 18 \\
\text { years or older with gestational age of } \\
20 \text { weeks or more and without } \\
\text { mental illness } \\
\text { Exclusion criteria: previous DM, } \\
\text { Gestational age }<24\end{array}$ & $\begin{array}{l}\text { Higher folate and potassium level in } \\
\text { the vitamin supplements increased } \\
\text { the risk of GDM. } \\
\text { Increased FA level aggravates the } \\
\text { effects of vitamin } \mathrm{B}_{12} \text { deficiency and } \\
\text { exacerbates insulin resistance. }\end{array}$ \\
\hline Cheng, G. & $\begin{array}{l}\text { 2019/China/ } \\
\text { Cohort }\end{array}$ & $\begin{array}{c}950(\text { GDM cases } \\
\text { were } 10.2 \%)\end{array}$ & $\begin{array}{l}\text { Inclusion criteria: infants had health } \\
\text { care records in the CHMIS; mothers } \\
\text { and infants' guardians agreed to } \\
\text { participate and signed the written } \\
\text { informed consents; and mothers took FA } \\
\text { supplements at a dose of } 400 \mathrm{mcg} / \mathrm{day} \\
\text { before, and during, pregnancy. } \\
\text { Exclusion criteria: mothers had a } \\
\text { history of mental illness or brain } \\
\text { disease, diabetes before pregnancy; } \\
\text { multiple pregnancy; congenital } \\
\text { abnormalities in the newborn }\end{array}$ & $\begin{array}{c}\text { FA supplementation for } \geq 3 \text { months } \\
\text { before pregnancy was associated } \\
\text { with an increased risk of GDM } \\
\text { (aRR: } 1.72 ; 95 \% \text { CI: } 1.17-2.53 \text { ) }\end{array}$ \\
\hline
\end{tabular}

After adjusting for confounding

$\begin{array}{ccc}\text { Huang, L. } & \text { 2019/China/ } & \text { 326: } 33(10.1 \%) \\ \text { Cohort } & \text { had GDM }\end{array}$

N/A factors, the aOR of GDM comparing taking folic acid for $>90$ days with taking folic acid for $\leq 60$ days was 3.45 (95\% CI: 1.01, 11.8)

Li, Q. $\quad \begin{aligned} & \text { 2019/China/ } \\ & \text { Cohort }\end{aligned}$
Exclusion criteria: those with diabetes, multiple pregnancies, abortions, without reliable OGTT or folic acid supplementation values
FA supplement use $>800 \mathrm{mg} /$ day from pre-pregnancy through mid-pregnancy was associated with the development of GDM

$\begin{array}{ccc}\text { Zhang, H. } & \text { 2019/China/ } & \text { 5165: } 604(11.7 \%) \\ \text { Cohort } & \text { had GDM }\end{array}$

Exclusion criteria: women with one of the following

Conditions: pre-existing type 1 and type 2 DM or GDM, thyroid disease, hypertension, asthma and systemic lupus erythematosus.
First trimester exposure to ai polluted with $\mathrm{SO}_{2}$ increased the risk of GDM, especially if between the $4^{\text {th }}$ and $10^{\text {th }}$ weeks of gestation, and if using FA supplements. $\begin{array}{ccc}\text { Zhu, B. } & \text { 2019/China/ } & \text { 1938: } 249(12.8 \%) \\ \text { Cohort } & \text { had GDM }\end{array}$
Inclusion criteria: Women who had either used FA supplements or never used any vitamin supplements
Higher risk of GDM was found with daily FA supplementation during the first trimester $(\mathrm{aOR}=2.25$ [95\% CI 1.35 - 3.76] $)$ 
Table S2. Excluded studies.

\begin{tabular}{ccc}
\hline Author's name & Study type/Country/Year & Reason for exclusion \\
\hline Kozlowska, A [26] & Prospetive/Poland/2018 & $\begin{array}{c}\text { This study focused on multivitamins, including folate. It did not focus in any section } \\
\text { on folate as an independent factor that can impact gestational diabetes risk }\end{array}$ \\
\hline Li, S [18] & Cross-sectional/China/2019 & $\begin{array}{c}\text { This study did not measure folate intake. Instead, it used serum folate level } \\
\text { to relate to the risk of developing gestational diabetes }\end{array}$ \\
\hline Barzilay, E [28] & Cohort/Canada/2018 & $\begin{array}{c}\text { This study measured serum concentrations of one-carbon } \\
\text { nutrients, not folate intake }\end{array}$ \\
\hline Lai, JS [16] & Cohort/Singapore/2018 & $\begin{array}{c}\text { This study tried to link serum concentrations of folate along with other } \\
\text { vitamins to the risk of gestational diabetes }\end{array}$ \\
Araujo, JR & Case-control/Portugal/2012 & $\begin{array}{c}\text { This study measured folate uptake by primary cultured cytotrophoblasts isolated } \\
\text { from human placentas of patients with gestational diabetes and compared } \\
\text { them to controls }\end{array}$ \\
Reece, EA & Review/USA/2012 & $\begin{array}{c}\text { This review did not relate folic acid supplementation to the development of } \\
\text { gestational diabetes, but it discussed the protective impact of folate on the } \\
\text { risk of neural tube defects }\end{array}$
\end{tabular}

